\title{
Study of Non-stationary Signal of Gearbox Based on Angle Domain Fourier Transform Analysis
}

\author{
Tian $\mathrm{Hao}^{1}$, Kang Xiao-yong ${ }^{1}$, Zhang Jun-nuo ${ }^{1}$, Wang Bao-bao ${ }^{2}$ \\ 1. Department of Guns Engineering, Ordnance Engineering College, Shijiazhuang, Hebei, 050003, \\ China \\ 2. No.77611 troop of PLA, Tibet, Lhasa, 850000, China \\ tgok@163.com
}

Keywords: gearbox, angle domain, Fourier transforms, rotating machinery.

\begin{abstract}
The processing method of the non-stationary rotary machine vibration signal is studied. The angle domain signal is resampled from the time domain signal with interpolation algorithm. The time domain averaging method is preformed on the resampled signal to depress the noise uncorrelated to the axial frequency. The cepstrum analysis technique is introduced into the order analysis method to identify the periodic information hiding in the power spectrum. The gear tooth crack fault is diagnosed successfully. It shows that the techniques above are suitable and effective for analyzing the signal of the rotary.
\end{abstract}

\section{Introduction}

For the stability of the mechanical power frequency, average can use ordinary spectrum analysis method based on FFT to extract the characteristic frequency effectively, diagnosed with its common faults. But many of the rotating machinery in the working process of the rotating speed is not in a state of absolute uniform, but changing within a certain speed range. Especially in the process of start and stop, its power frequency is not stable. If artificially will assume that this kind of signal is stationary signal processing, the results will have a serious phenomenon of frequency fuzzy[1]. In order to solve this problem, order tracking theory arises at the historic moment.

Engineering testing signal often contains a lot of noise components, so on the analysis of the periodic steady-state signal data average method is often used to suppress noise to improve signal-to-noise ratio[2]. For rotating machinery, this paper, we assume that the Angle of system speed changes in the frequency domain is only for the signal amplitude effect without affecting its frequency, based on the diagonal domain resampling signals the Angle domain average. Analysis of example proved the feasibility of this method.

Rotating machinery vibration signal is complex, only through the spectrum analysis is not enough. Due to the inverse spectrum into a family of the spectrum can be simplified to single side band spectral lines of the spectral lines, can detect the power spectrum is difficult to identify the periodic, so this article will fall frequency analysis theory is introduced into order time, in order to get the signal, more order spectrum to extract fault features.

\section{The realization of the inverted order spectrum analysis}

\subsection{Calculated order analysis}

Calculated the order analysis method is an advanced technology developed in recent years, its purpose lies in through the signal processing algorithm will wait time interval sampling signal is converted into the perspective of sampling signals, namely, synchronous sampling signal. Compared with the hardware order analysis method, this method can reduce the cost, ease restrictions on sensor installation and can improve the Angle domain sampling rate, advantage is obvious. The specific implementation process is as follows. 
Usually, in order to determine the time point of resampling, should first set a reference axis Angle acceleration model, it is generally believed in a small period of reference shaft Angle of uniformly accelerated motion, forefathers' research[3] had prove the feasibility of this assumption.

Angle can be calculated according to the type of sampling at a constant Angle increment corresponding to the needs of the interpolation point in time.

According to request a point in time, using the interpolation algorithm for interpolation, the vibration signals can be calculated vibration signal in the angular domain amplitude corresponds to the sampling time point, again carries on the FFT transform, the result is order spectra after resampling.

In particular, the above algorithm still needs to meet the sampling theorem, it displays in two aspects: first, the resampling the sampling rate of an unlimited increase, should be affected by time domain sampling signal sampling rate; Second, can't analysis order times higher than half the sampling rate of the signal component, lest produce mixed up.

\subsection{Angle domain average algorithm}

Average method is often used to analyze the multiple periodic steady-state signal, in order to weaken the influence of noise, improve signal-to-noise ratio, its principle is as follows

For rotating machinery, as a result of the changes in speed signal amplitude also corresponding change, even for isometric domain sampling after its signals is still not satisfied periodic steady-state conditions, but according to the mechanical vibration knowledge it is easy to know the system each roll a cycle of power frequency related impact frequency is fixed, fault impact as well. So, might as well, we assume that the Angle of system speed changes in the frequency domain is only for the signal amplitude effect without affecting its frequency. So we can still adopt the way of average improve signal-to-noise ratio.

\subsection{Cepstrum analysis}

Cepstrum defined as: the power spectrum of Fourier inverse transformation was carried out on the numerical results.

Inverse spectrum on the spectrum into side band spectral lines of simplified to single line, can detect the power spectrum is difficult to identify the cyclical. When mechanical failure to produce a periodic signal changes, inverse spectrum will appear the corresponding peak, inverse spectrum pulse index reflects the degree of the change.

Because the order analysis is the time domain signal resampling to Angle domain signal, the signal expression changed, only the essence has not changed, so similar with the traditional spectrum analysis, when the fault signal and the system normal signal phase modulation, in order spectrum is bound to produce the corresponding edge band. In this paper, the order cepstrum analysis algorithm is introduced into the analysis, in order to order sideband is simplified to single line, to identify mechanical failure.

\section{Analysis of experiment}

Experiment with a speed reducer, and adjustable speed motor input, the driving gear teeth $\mathrm{Z} 1=30$, passive gear teeth $\mathrm{Z} 2=50$. In does not affect the bearing under normal use performance, in a driving gear tooth root processing $0.5 \mathrm{~mm}$ wide, deep $1.5 \mathrm{~mm}$ small tank simulation root crack. Through BK3560 analyzer to collect more speed signal and vibration acceleration sensor signal photoelectric encoder, the rising process of gear box are analyzed.

Figure 1 as the input shaft speed curve, the figure is the input shaft from zero to 700 RPM, accelerated motion, namely the system power frequency instability. Figure 2 for angular domain re-sampling signals, the figure shown with the increasing of rotation speed and vibration signal enhancement, for non-stationary signal. Figure 3 for the three power frequency cycle length for the cycle after the Angle domain average sampling signal. Although many times the average, signal amplitude change is still very obvious. 


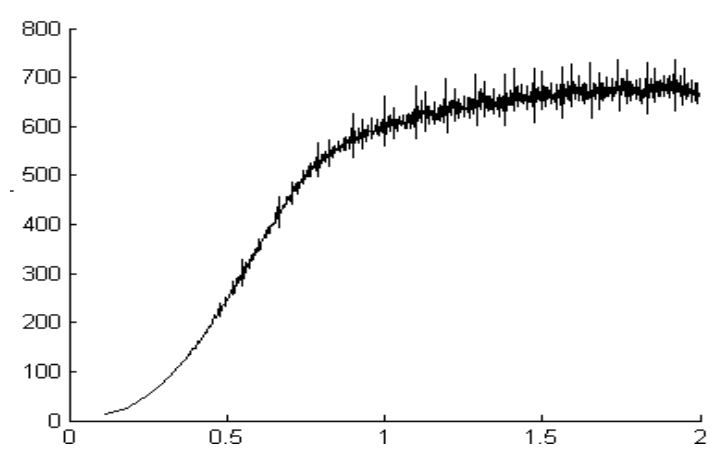

Fig.1 speed curve

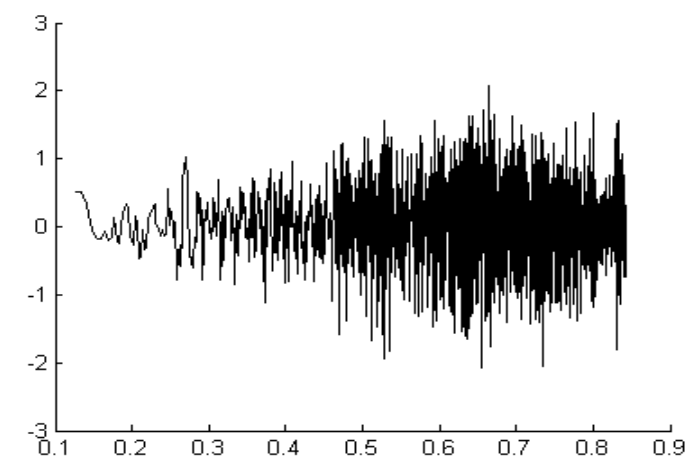

Fig.3 resampling signal after angle domain average

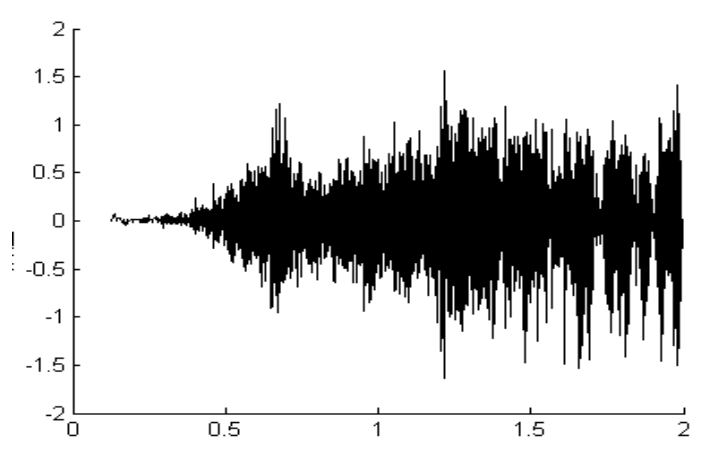

Fig.2 resampling signal

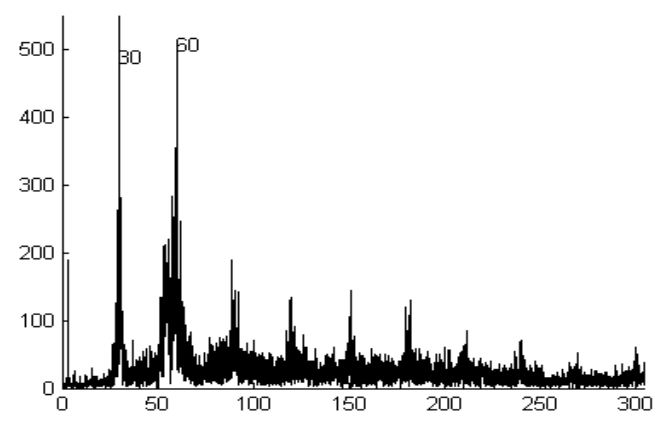

Fig.4 order spectrum of resampling signal
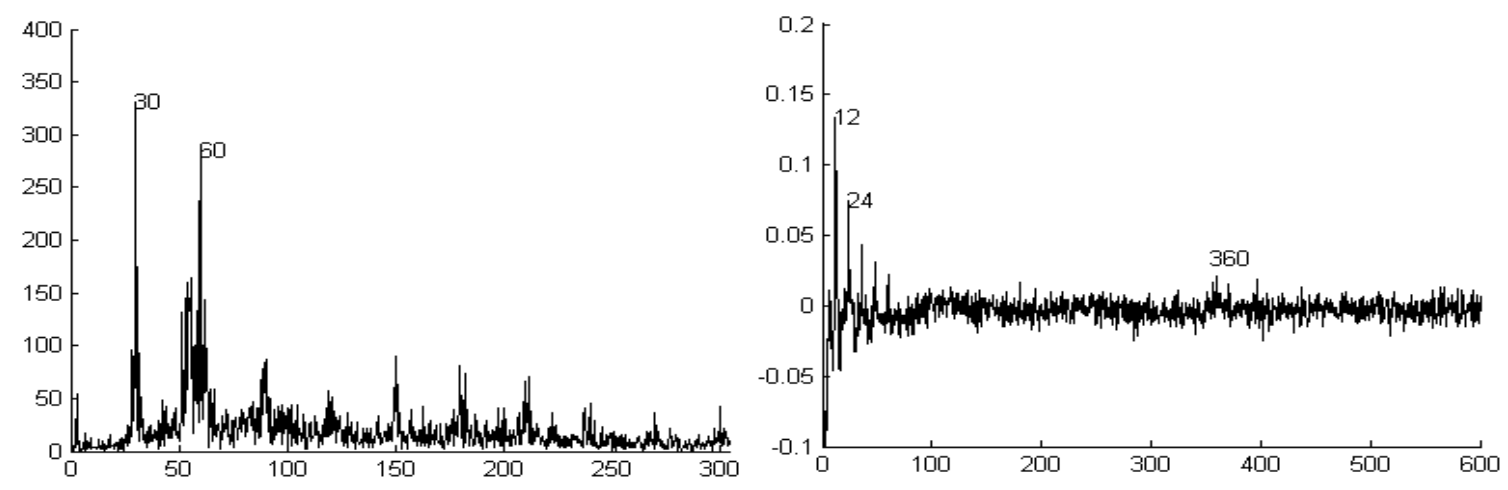

Fig.5 order spectrum of resampling signal after angle domain average Fig.6 Inverted order spectrum of resampling signal

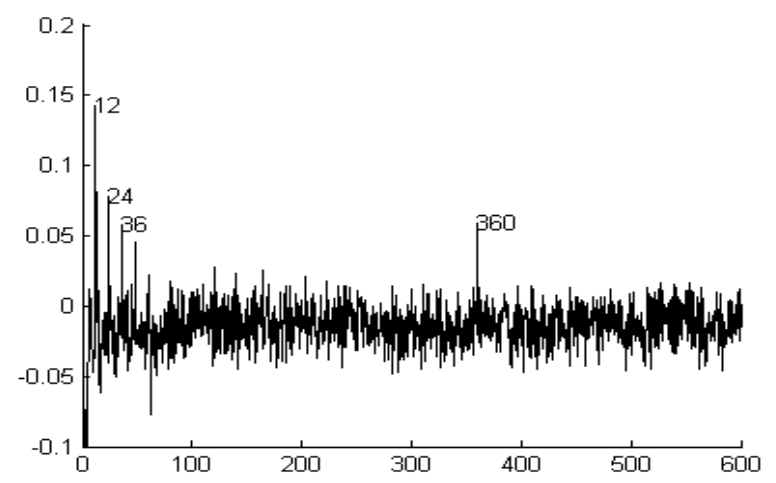

Fig.7 Inverted order spectrum of resampling signal after angle domain average

Figure 4 a major order of sampling signal spectrum, the figure shown clear mesh order time and the frequency doubling, description order time away from the traditional spectrum analysis on the analysis of the variable speed signal frequency mixing overlapping phenomenon. Figure 5 for seed domain average after order spectrum signal. By the chart shows, the former than the latter line, obviously it contained more noise components. Due to the effect of tooth of frequency modulation, tooth root crack fault characteristics reflected in the side band, so no obvious reflected in the above two figure. The need for further processing. 
Figure 6 major spectrum sampling signal of the inverted order time. Figure in the meshes of frequency inverted order $\left(12^{\circ}\right)$ and the frequency doubling is very obvious, but the root crack fault feature order $\left(360^{\circ}\right)$ is not significant. Figure 7 to Angle domain average after order spectrum. Diagram meshes frequency information clearly, and root crack fault characteristics of the order is clearly visible. For this type of signal is to verify the failure state, illustrates the feasibility and effectiveness of Angle domain average.

\section{Conclusion}

On the analysis of rotating machinery unsteady signal, the traditional spectrum analysis method for frequency of "fuzzy" and couldn't reflect the true state of the system. Order analysis method based on power frequency, using the perspective of sampling signal were analyzed, and meet the requirements of the FFT transform of signal stability, the speed signal processing, can effectively solve the frequency of "fuzzy" phenomenon, is a powerful complement to the traditional spectrum analysis. Because there is also in the Angle domain signal modulation phenomenon, only through the order analysis to determine the state of the system often cannot achieve the desired effect, so for accurate diagnosis system failures still need further processing; Inverted order spectrum analysis is more effective in the diagnosis of the gearbox fault. For rotating machinery, this paper, we assume that the Angle of system speed changes in the frequency domain is only for the signal amplitude effect without affecting its frequency, based on the diagonal domain resampling signals the Angle domain average. The analysis demonstrated the feasibility and effectiveness of the method.

\section{References}

[1] FYFE K R, MUNCKEDS. Analysis of computed order tracking[J].Mechanical Systems and Signal Processing, 1997,11(2):187-205.

[2] Kim J T, Lyon R H. Cepstrum analysis as a tool for robust processing, reverberation and detection of transients[J]. Mechanical System and Signal Processing, 1992,6(1):1-15.

[3]Li Xiaohu, Jia Minping. Spectrum Analysis and Its Application to Gearbox Fault Diagnosis[J].Journal of Vibration,Measurement\&Diagnosis,2003,23(3):168-170

[4]Gao Xu, Huang Wei. Diagnosis Method of Gear Worn Fault[J].Journal of Anshan Institute of I.\&S. Technology,2000,23(6):453-456.

[5]Cheng Hang, Cheng Minghuang. The Application of Cepstrum in Gear Fault Diagnosis[J].JOURNAL OF TAIYUAN UNIVERSITY OF TECHNOLOGY,2003,34(6): 661-663

[6] Zhang Han-lei. Compound-fault diagnosis of induction motor based on wavelet anaylsis[J]. Transaction of Chinese Electric Engineering, 2006, 26 (8):159-162.

[7] Feng Zhi-peng. Overview of rotary mechanical vibration fault diagnosis theory and technic[J]. Journal of Vibration and Shock,2001, 20 (4):36-39.

[8] Zhu Ji-mei. Unsteady vibration signal analysis[J]. Journal of Vibration and Shock. 2000,19(2):87-90.

[9] Tang You-huai. Electric engine fault diagnosis based on wavelet packet energy spectrum analysis[J]. Foreign electronic surveying technic, 2008,27(2):54-63 